\title{
EFFECTS OF AQUEOUS EXTRACT OF GLYCYRRHIZA GLABRA LINN. AND DIOSMETIN ON MODULATION OF SPATIAL MEMORY THROUGH ACETYLCHOLINESTERASE AND BRAIN-DERIVED NEUROTROPHIC FACTOR IN ETHANOL-INDUCED COGNITIVE IMPAIRMENT MODEL RATS
}

\author{
SASIKUMAR ${ }^{1}{ }^{1}$, SUBA MALANI $S^{1}$, MANIKANDAN $\mathbf{S}^{1 *}$, RAMASWAMY $\mathbf{C}^{2}$, SENTHILKUMAR $\mathbf{S}^{3}$ \\ ${ }^{1}$ Department of Physiology, Tagore Medical College and Hospital, Rathinamangalam, Chennai, Tamil Nadu, Affiliated to The TN Dr. MGR \\ Medical University, Chennai, Tamil Nadu, India. ${ }^{2}$ Department of Physiology, Saveetha Medical College and Hospital, Saveetha Institute of \\ Medical and Technical Sciences, Saveetha Nagar, Thandalam, Kancheepuram, Chennai, Tamil Nadu, India. ${ }^{3}$ Department of Research and \\ Development, Saveetha Institute of Medical and Technical Sciences, Chennai, Tamil Nadu, India.
}

Email: drsmanikandan@yahoo.com

Received: 20 June 2018, Revised and Accepted: 20 August 2018

ABSTRACT

Objective: The objective of this research was to evaluate the cognitive impairment due to excessive consumption of alcohol and memory enhancement action of Glycyrrhiza glabra Linn. (AEGGL) and diosmetin (Dm).

Methods: In this study, 36 adult male Wistar rats were divided into the six groups $(n=6)$ and eight-arm radial maze, narrow beam test, and open field behavior parameters were assessed on day 1, 10, and 21. After the 21 days of experiment, animals were sacrificed, and blood samples were collected for serum acetylcholinesterase (AChE) and brain-derived neurotrophic factor (BDNF) estimation. We have also analyzed the morphology of CA3 region of the hippocampus.

Results: The results of this study suggested that AEGGL and Dm treatment could be the potential drugs for ethanol-induced cognitive impairment.

Conclusion: Ethanol-induced cognitive impairment was recovered by AEGGL and Dm treatment, we suggested that this might be due to anticholinesterase activity and increased synthesis of BDNF levels in the brain. Further, researches are warranted to understand the exact mechanism of action of drugs.

Keywords: Glycyrrhiza glabra Linn., Diosmetin, Ethanol, Cognitive impairment, Spatial memory.

(C) 2018 The Authors. Published by Innovare Academic Sciences Pvt Ltd. This is an open access article under the CC BY license (http://creativecommons. org/licenses/by/4. 0/) DOI: http://dx.doi.org/10.22159/ajpcr.2018.v11i12.27992

\section{INTRODUCTION}

Alcohol is one of the most consumed products and becomes a trend among the youngsters in India. The World Health Organization has reported that Indians reached the second place in South East Asian Region in alcohol consumption [1]. Excessive exposure to alcohol leads nervous system-related diseases with or without nutritional deficiencies. Metabolism and toxic effects of ethanol vary in the brain region and depend on age, dosage, and duration of consumption. However, recent studies show that alcohol affects neuronal cells, specifically astrocytes and microglial cells are the major targets. This leads to white matter atrophy, neuroinflammation, and impaired neurogenesis [2]. Catalysis of alcohol produces reactive oxygen species ends up with oxidative stress and results in many irreversible changes in the brain [3]. Cognitive abilities are fully depend on the hippocampus structure integrity and neurotransmitter acetylcholine (ACh) levels. Acetylcholinesterase (AChE) is considered as treatment target to keep up the ACh level in some neurodegenerative disorders such as Alzheimer's disease and dementia [4]. Accumulative evidence have demonstrated that ethanol caused cognitive impairment and cholinergic dysfunction associated with oxidative stress [5,6]. Longterm cognitive deficits, alteration in-memory processing, and changes in synaptic transmission were associated with acute and chronic alcoholic models through action on glutamate, gamma-aminobutyric acid, and N-methyl-D-aspartate receptors (NMDAR) [7]. Ethanolinduced mild cognitive impairment (MCI) was produced at the dose of $1.75 \mathrm{mg} / \mathrm{kg}$ of ethanol for 21 days to identify the better cholinesterase inhibitors between donepezil and rivastigmine for treating cognitive deficits [8]. Neuroprotective effects of neurotrophins are playing key role in modulation of cognitive impairment and functional recovery after brain tissue inflammation. Especially, brain-derived neurotrophic factor (BDNF) involved with neuronal survival and plasticity which is synthesized primarily from neurons secondarily from neuroglial cells of inflamed brain tissue [9]. Elevated BDNF levels were ameliorating the cognitive impairment by supporting the injured hippocampus. Estimation of BDNF exhibits the neuronal integrity of CA3 pyramidal neurons of hippocampus [10].

Currently, very few approved drugs are available for cognitive impairment and neurodegenerative disorders caused by alcoholism because of less bioavailability and adverse side effects [11]. Therapeutic benefits of natural products were considered in drug discovery field to the find better molecule for neurological disorders. Glycyrrhiza glabra Linn. (GGL) is a medicinal plant commonly known as licorice (Fabaceae family) and athimadhuram in Tamil which has many therapeutic potential to treat as hepatoprotective, antiulcerative, and antimicrobial agents [12]. Enhancement action of GGL (AEGGL) is one of the herbal extracts, commonly used Indian system of medicine for enhancing learning and memory [13]. AEGGL has lethal dose as higher that can be used for chronic consumption [14]. Dm is known antioxidant molecule which is responsible for the protective effect against neurotoxicity [15]. Dm produced improvement of spatial learning, working memory and represents potential therapeutic approaches for the treatment of cognitive impairments in persons with neurocognitive disorders [16]. These natural products possessing the antioxidant activity against the ethanol-induced neurotoxicity has been considered to improve the cognitive impairment. 
Thus, this study was aimed to investigate the effects of AEGGL and Dm on spatial memory, cholinergic function, and motor activities of Wistar rats with ethanol-induced cognitive impairment.

\section{METHODS}

\section{Experimental animals}

A total of 36 adult male Wistar albino rats (Rattus norvegicus), with 180$280 \mathrm{~g}$ body weight were purchased from Center for Laboratory Animal Research, Department of Research Development, Saveetha Institute of Medical and Technical Sciences, Chennai. This study was approved by IAEC Reference number - (SU/CLAR/RD/038/2017) dated 25/08/17. The rats were housed in polypropylene cages with paddy husk bedding, standard food pellets, and drinking water ad libitum, and $12 \mathrm{~h}$ light and $12 \mathrm{~h}$ dark schedule in $23^{\circ} \mathrm{C} \pm 2^{\circ} \mathrm{C}$ were provided.

\section{Chemicals and drugs}

Ethanol (95\%, Nanda, Inc.), normal saline, diosmetin (DM) (Sigma, Inc.), AChE (Sigma, Inc.) and BDNF assay kit (Bosterbio, USA) dimethyl sulfoxide (DMSO), and other laboratory chemicals (Labthi, Inc.) were purchased for this experiment.

\section{Plant material and extract preparation}

The roots of GGL were purchased from local herbal market in Chennai and got authenticated from medicinal botanist, Department of Medicinal Botany, National Institute of Siddha, Tamil Nadu, India. The specimen was deposited for future reference, and the authentication reference number is NIAS 1502015.

The roots were dried and powdered to mix with distilled water in Soxhlet apparatus for aqueous extract preparation. The filtrate was collected and evaporated using reduced pressure evaporator, and the extract yield was collected for the study [17].

\section{Experimental groups}

- Control group received DMSO for 21 days intragastrically (i.g).

- ETOH group received ETOH $(1.75 \mathrm{mg} / \mathrm{kg} / \mathrm{d})$ for 21 days (i.g).

- AEGGL group received AEGGL (150 mg/kg/d) along with DMSO for 21 days (i.g).

- Dm group received Dm (4 mg/kg/d) along with DMSO (1 mg/kg/d) for 21 days (i.g).

- AEGGL+EtOH group received AEGGL $(150 \mathrm{mg} / \mathrm{kg} / \mathrm{d})$ along with ethanol $(1 \mathrm{mg} / \mathrm{kg} / \mathrm{d})$ for 21 days (i.g).

- Dm+EtOH group received Dm (4 mg/kg/d) along with ethanol (1.75 mg/kg/d) for 21 days (i.g).

\section{Standardization of behavioral tests}

All animals were transferred to experimental room from quarantine room, after acclimatization behavioral training was given for 1 week. In this period, animals were familiarized with apparatus and test procedures. During behavioral training period standardize the experimental procedure was done. Behavioral assessments were studied using open field test (OFT), eight-arm radial maze (EARM), and narrow beam test. Behavioral studies were conducted on the $1^{\text {st }}$, $10^{\text {th }}$, and $21^{\text {st }}$ day of the experiment for six groups after $4 \mathrm{~h}$ of drug administration.

\section{EARM}

The radial arm maze consists of eight arms, numbered from 1 to 8 arms of $48 \mathrm{~cm} \times 3 \mathrm{~cm} \times 12 \mathrm{~cm}$, extending radially from a central platform ( $40 \mathrm{~cm}$ in diameter), with a $5 \mathrm{~cm}$ edge around the apparatus. Each radial arm is equally spaced and contains food cups at the end. Removable blocks of $9 \mathrm{~cm} \times 3 \mathrm{~cm} \times 13 \mathrm{~cm}$ were used to block the selected arm of the maze. The maze was elevated $40 \mathrm{~cm}$ from the ground or floor. The following variables were scored for 5 min and recorded: Number of reference memory errors (entry of animal into the non-baited arm), number of working memory errors (reentry of animal into already visited baited arm), and time taken to visit all four baited arm latency [18].
OFT

OFT is a measure of emotional behavior in animal. It is a systematic assessment of new environment exploration, locomotor activity, and anxiety-related behaviors in rats. The OFT apparatus is made of a large square-shaped arena of $80 \mathrm{~cm} \times 80 \mathrm{~cm}$ with $40 \mathrm{~cm}$ high walls. The floor is marked into 25 equal square segments to allow quantification of locomotor activity. Each rat was placed at the center of the arena and was observed the time spent in the periphery of the arena, time spent in the center of the arena, number of squares (NOS) crossed (NSC) by the animals, and number of fecal pellets passed [19].

\section{Narrow beam test}

The rats were trained to walk on a $10-20 \mathrm{~mm}$ wide wooden beam elevated $100 \mathrm{~cm}$ above the floor. After training, traversing time through beam to reach safe platform (100 mm distance) and NOS was quantified [20].

\section{Blood sample collection and tissue preparation}

After behavioral assessment, animals were anesthetized with isoflurane and blood was collected for serum BDNF and AChE estimation. Estimation of acetylcholinesterase and BDNF level in plasma was done as per manufacturer instruction $[21,22]$. The animals were sacrificed and brain tissue was harvested and placed forma buffered solution for $48 \mathrm{~h}$ for dehydration. The dehydrated brain was washed with cold saline and embedded with paraffin wax. The blocks were sectioned as $3 \mu \mathrm{m}$ thickness by microtome at levels of hippocampus in the rat brain. The sections were mounted on glass slides and left for dry in hot plate $\left(40^{\circ} \mathrm{C}\right)$. The slides were dewaxed using xylene followed by hematoxylin and eosin staining, and sections were used to count the number of neuron by trinocular microscope (Olympus-BX40) and image J2 software [23].

\section{Statistical analysis}

The data were expressed as mean \pm SEM and analyzed using one-way analysis of variance followed by post hoc Tukey's test for multiple comparisons of groups. All statistical analyses and graph plotting were carried out using SPSS software 22.0 (Chicago, USA). $p<0.05$ is considered as statistically significant.

\section{RESULTS}

\section{Effect of AEGGL and Dm on spatial memory}

Figs. 1-3 showed that progressive reduction of spatial memory as decreased latency period and increased working methanolic extract (WME) and reference methanolic extract (RME) in EARM test in ETOH group in time-dependent manner. AEGGL+EtOH group and Dm+ EtOH group have shown significant improvement in spatial memory compared with ETOH group as the effect of drugs given for 21 days. Comparing the results in between groups by Tukey's post hoc analysis on the $21^{\text {st }}$ day, WME $(\mathrm{F}=20.429, \mathrm{p}<0.001)$, RME $(\mathrm{F}=20.078, \mathrm{p}<0.001)$, and latency period $(\mathrm{F}=27.889, \mathrm{p}<0.001)$ were statistically significant. Drug only treated groups such as AEGGL and Dm group was shown less WME, RME, and increased the latency period than control group after 21 days $(F=2.543, p<0.05)$. Specifically, AEGGL group has shown significant effects on ETOH-induced MCI rats than Dm group after the $10^{\text {th }}$ and $21^{\text {st }}$ day.

\section{Effect of AEGGL and Dm on open field exploration}

The anxious behavior of animals in OFT such as time spent in center of arena, near peripheral walls, and fecal pellet was showed in Figs. 4-7. ETOH group spent more time in periphery as day progressively than control group, AEGGL and Dm group after the $10^{\text {th }}(\mathrm{F}=12.184, \mathrm{p}<0.001)$ and $21^{\text {st }}$ day $(\mathrm{F}=24.291, \mathrm{p}<0.001)$. AEGGL $+\mathrm{EtOH}$ group and $\mathrm{Dm}+\mathrm{EtOH}$ spent more time in center of arena than ETOH group. Ethanol reduces the NOS crossing in OFT in a day-dependent manner that AEGGL and Dm groups showed significant ambulation than control group on the $10^{\text {th }}$ day $(\mathrm{F}=24.943, \mathrm{p}<0.001)$ and $21^{\text {st }}$ day $(\mathrm{F}=32.089, \mathrm{p}<0.01)$. AEGGL $+\mathrm{EtOH}$ and Dm+ EtOH group exhibit the significant difference in NSC than the ETOH group that drugs improve the ambulation. 
Effect of AEGGL and Dm on ambulation

Figs. 8 and 9 demonstrated that the effects of AEGGL and Dm on latency to cross the narrow beam and NOS during the traverse. The results showed that the motor activity is affected in ETOH group than AEGGL+EtOH and Dm+EtOH groups throughout the days statistically $(\mathrm{F}=2.018, \mathrm{p}<0.04)$. NOS were significant in control, AEGGL and Dm treated groups than ETOH group after 21 days of experiment. NOS were

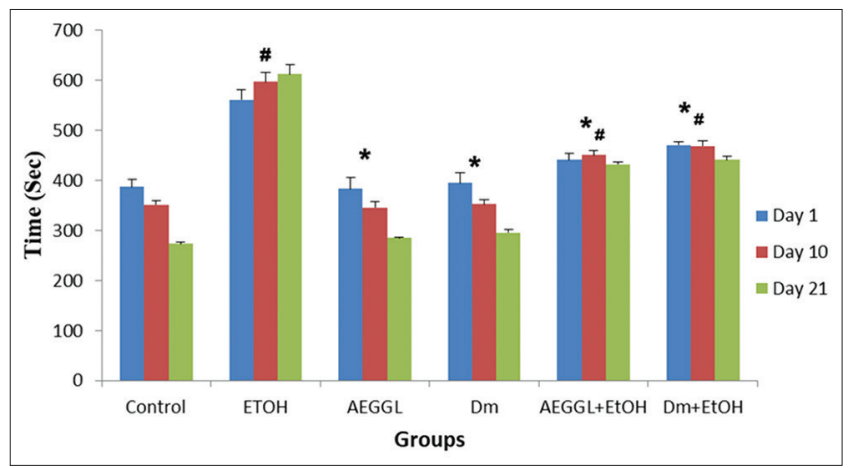

Fig. 1: Effects of enhancement action of Glycyrrhiza glabra Linn. and diosmetin on latency periods in eight-arm radial maze test. Data were expressed as mean \pm standard error mean and analyzed by one-way analysis of variance followed by post hoc

Tukey's test for multiple comparison of groups $(n=6)$. \# is significant from control and *is significant from ETOH

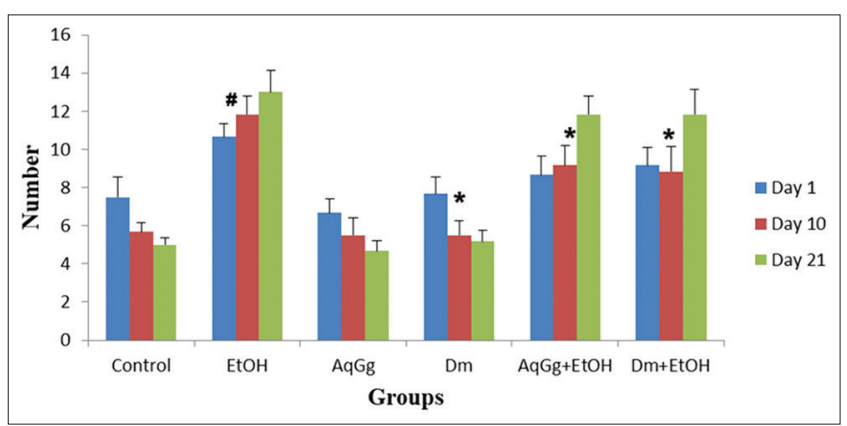

Fig. 2: Effects of enhancement action of Glycyrrhiza glabra Linn. and diosmetin on working methanolic extract in eight-arm radial maze test. Data were expressed as mean \pm standard error mean and analyzed by one-way analysis of variance followed by post hoc Tukey's test for multiple comparison of groups $(n=6)$. \#is significant from control and *is significant from ETOH

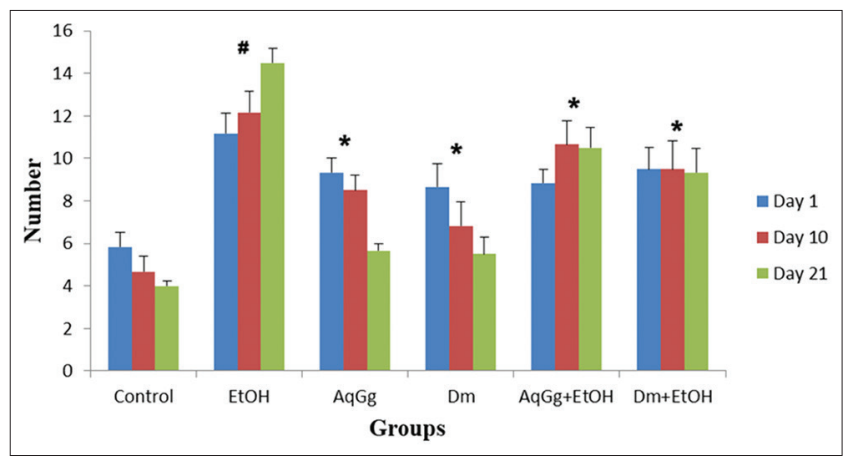

Fig. 3: Effects of enhancement action of Glycyrrhiza glabra Linn. and diosmetin on reference methanolic extract in eight-arm radial maze test. Data were expressed as mean \pm standard error mean and analyzed by one-way analysis of variance followed by post hoc Tukey's test for multiple comparison of groups $(n=6)$. \#is significant from control and ${ }^{*}$ is significant from ETOH prevented in AEGGL+EtOH and Dm+EtOH group $(\mathrm{F}=2.265, \mathrm{p}<0.05)$ than ETOH group as showed the effect of drugs on motor activities in time-dependent manner.

Effect of AEGGL and Dm on AChE activity and BDNF levels

Since cholinergic neurons played a key role on spatial memory, we also investigated the effect of AEGGL and Dm on AChE activity in the plasma, and the results were shown in Table 1 . The activity of AChE

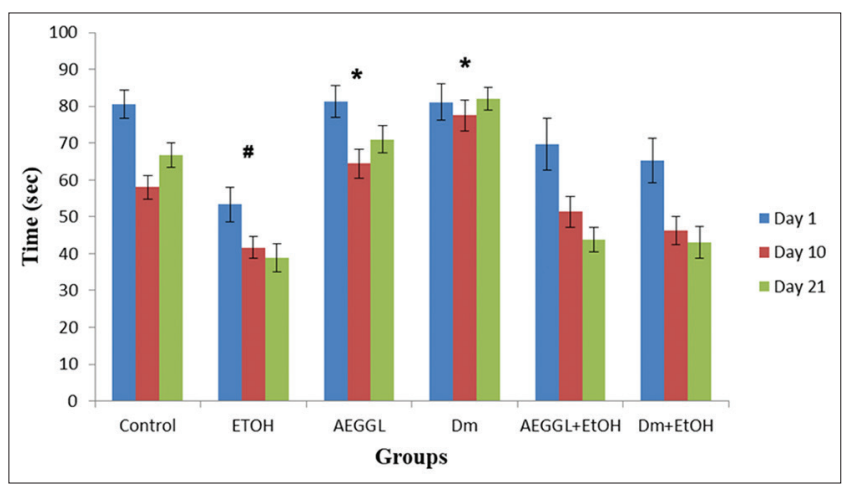

Fig. 4: Effects of enhancement action of Glycyrrhiza glabra Linn. and diosmetin on time spent in center in open field test. Data were expressed as mean \pm standard error mean and analyzed by one-way analysis of variance followed by post hoc Tukey's test for multiple comparison of groups $(n=6)$. \#is significant from control and *is significant from ETOH

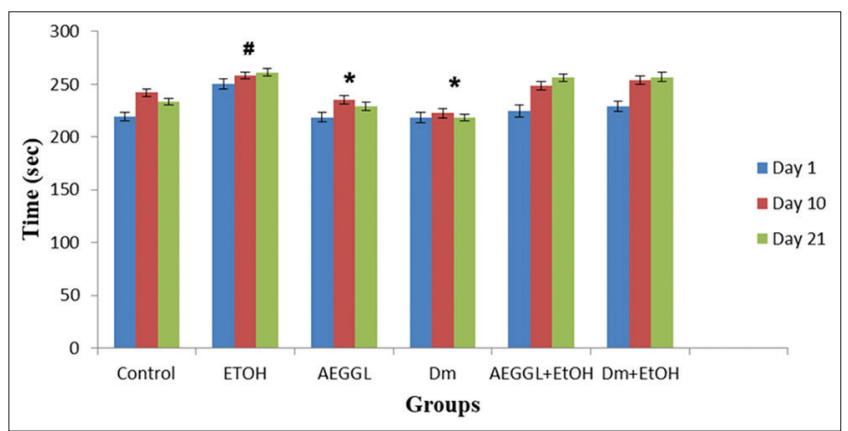

Fig. 5: Effects of enhancement action of Glycyrrhiza glabra Linn. and diosmetin on time spent in periphery in open field test. Data were expressed as mean \pm standard error mean and analyzed by one-way analysis of variance followed by post hoc Tukey's test for multiple comparison of groups $(n=6)$. \#is significant from control and *is significant from ETOH

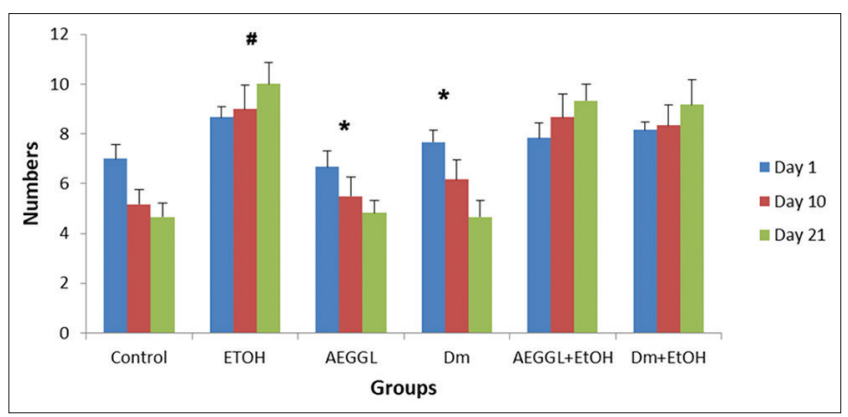

Fig. 6: Effects of enhancement action of Glycyrrhiza glabra Linn. and diosmetin on number of fecal pellets in open field test. Data were expressed as mean \pm standard error mean and analyzed by one-way analysis of variance followed by post hoc Tukey's test for multiple comparison of groups $(n=6)$. \#is significant from control and *is significant from ETOH 


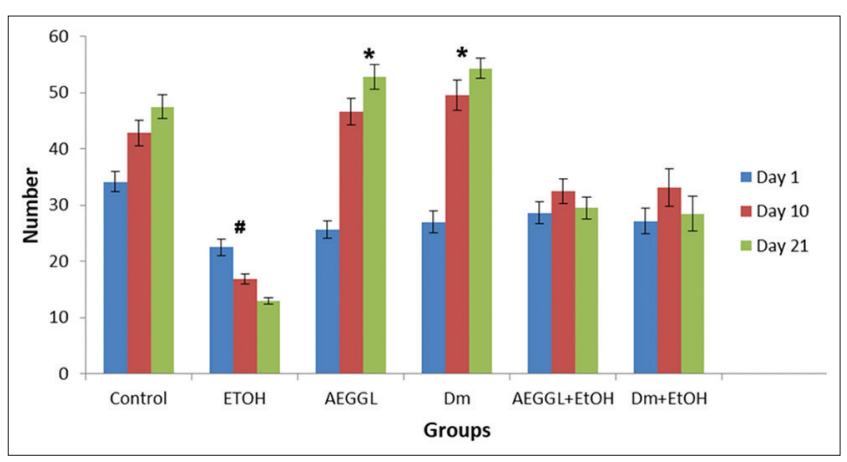

Fig. 7: Effects of enhancement action of Glycyrrhiza glabra Linn. and diosmetin on number of squares crossed in open field test. Data were expressed as mean \pm standard error mean and analyzed by one-way analysis of variance followed by post hoc

Tukey's test for multiple comparison of groups $(n=6)$. \#is significant from control and *is significant from ETOH

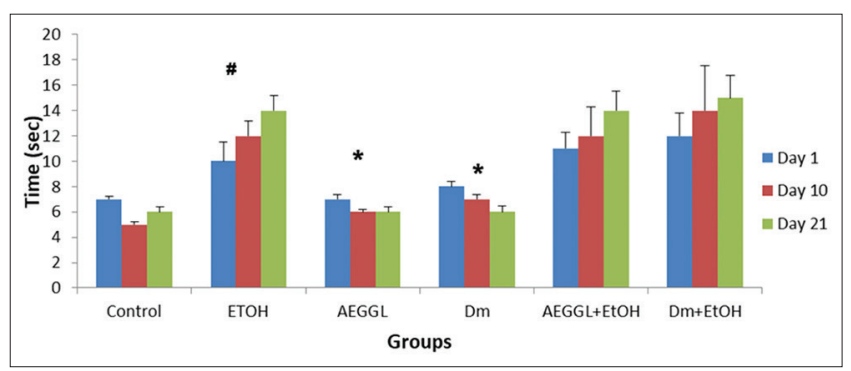

Fig. 8: Effects of enhancement action of Glycyrrhiza glabra Linn. and diosmetin on latency period in narrow beam test. Data were expressed as mean \pm standard error mean and analyzed by oneway analysis of variance followed by post hoc Tukey's test for multiple comparison of groups $(n=6)$. \#is significant from control and "is significant from ETOH

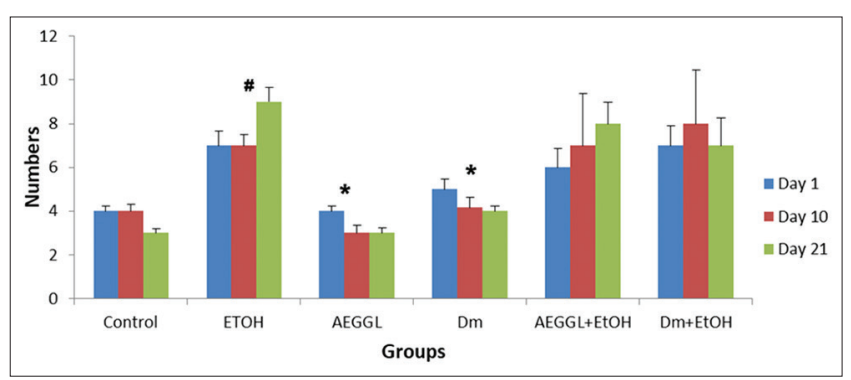

Fig. 9: Effects of enhancement action of Glycyrrhiza glabra Linn. and diosmetin on number of slips period in narrow beam test. Data were expressed as mean \pm standard error mean and analyzed by one-way analysis of variance followed by post hoc

Tukey's test for multiple comparison of group $(n=6)$ s. \#is significant from control and "is significant from ETOH

was expressed for as micromoles per minute milliliter of plasma. The significant reduction of AChE activity was observed in AEGGL+EtOH and $\mathrm{Dm}+\mathrm{EtOH}$ treated animals which maintain the cholinergic neurons after 21 days of experiment. Interestingly, AEGGL and Dm only treated groups were shown less AChE activity $(\mathrm{p}<0.001)$, compared with control rats which mean they could act as cholinesterase inhibitors. Overall, the results suggest that the AchE level was increased by ethanol induction $(\mathrm{F}=14.208, \mathrm{p}<0.001)$, and the drug components were used tried to bring back the ACh level to normal by decreasing the ACh esterase level.

Table 1 demonstrated that the effects of AEGGL and Dm on biochemical parameters. We have investigated the plasma BDNF level that was high in drug only treated groups compared with control group. Plasma BDNF level is less in ETOH group than AEGGL+EtOH and Dm+EtOH group significantly $(F=39.503, p<0.001)$ as shown as the beneficial effect of drugs. It shows that AEGGL increases the BDNF level than Dm that can be potential drug to manage the neuronal damage and increases the cognitive abilities after 21 days of ethanol induction.

\section{Effect of AEGGL and Dm on hippocampal pyramidal neurons}

Histological sections divulged the hippocampal pyramidal neurons in the CA3 region in each group (Fig. 4a-g). The average number of neurons in the CA3 region decreased and scattered layer was noticed in ETOH group (Fig. 4b) than control group. Ethanol ensues the decreased number of neurons in the AEGGL+ETOH (Fig. 4e and g) group and Dm+ETOH group (Fig. 4f) than control group (Fig. 4a); irregularities of neuronal layer were reduced from ETOH group. Normal pyramidal neurons were increased in AEGGL (Fig. 4c and g) and Dm group (Fig. 4d and g) than the control group. These findings suggest that AEGGL and Dm can be the potent drugs for ethanol-induced MCI (Fig. 10).

\section{DISCUSSION}

Many people have MCI by various causes, but identification and diagnostic aspects of cognitive dysfunction are difficult part, especially alcohol consumers. Awareness about ethanol-induced MCI and appropriate treatment strategies is required without adverse effects. We have observed the results clearly that the rats with ethanol-induced MCI advocate impaired spatial memory, locomotion, elevated AChE activity, and decreased plasma BDNF level. AEGGL and Dm protect the cognitive abilities from 21 days of ethanol neurotoxicity. In brief, rats were trained to navigate the EARM, OFT, and narrow beam test for 1 week, then $1.75 \mathrm{mg} / \mathrm{kg}$ ETOH given to the concern group from day 1 followed by drugs. Reversal of spatial memory impairment was induced by cerebral ischemia in rats assessed by EARM suggests that it is an ideal tool to confirm the cognitive functions specifically after impairment [24]. ETOH group has shown increased WME and RME after 10 days of ethanol exposure, which might be acute effects ETOH on orbitofrontal cortex. This may contribute to increased impulsivity and poor decision-making associated with subinducing levels of ethanol $[25,26]$. AEGGL and Dm were compensated the effects of ETOH and protect the cognitive abilities that might be enhanced by inhibition of AChE and activation of nicotinic and muscarinic cholinergic receptors. This activation enhances hippocampal excitatory input from entorhinal cortex and from dentate gyrus [27,28]. Histopathology of hippocampus exhibits that total number neurons were increased AEGGL and Dm group than control. Preserved neural layer disorganization and increased number of pyramidal neurons in the AEGGL+EtOH and Dm+ETOH groups that show the reduced neuroinflammation in the hippocampus and promote the learning memory functions [29]. OFT may be sensitive to hippocampal subregions pathways [30] and therefore increased open field activity has been used as an index of locomotor, anxiety, and exploratory activity in ethanol-induced rats. There was significant difference exist among groups in time spending in the center of the arena, rearing, and grooming behaviors of the test field. This decreased open field exploration in ethanol treated groups might be caused by NMDAR-dependent long-term depression in the hippocampus [31]. Alteration in the ion channels, enzymes, and receptors contributes into change in the synaptic functions by ETOH [32]. AEGGL and Dm might be act on receptor and ion channels at molecular level to protect from chronic ethanolic effects. The lethal dose 50 (LD50) of AEGGL was observed at the dose of $833.3 \mathrm{mg} / \mathrm{kg}$ body weight [14]. This might be an advantage to treat patients in dosedependent manner. However, the cognitive skills were enhanced by AEGGL and Dm, but motor activity did not much affect after 21 days while comparing with ETOH-induced groups in narrow beam test. Only acute effects of ethanol could be seen in the results. The concentration of consuming ethanol, duration of consumption, age, and other factors may disturb the locomotion. In this study, dosage of ethanol may cause only cognitive impairment and not motor disturbances [8]. The results of the current study show that 21 days ethanol administration impaired 


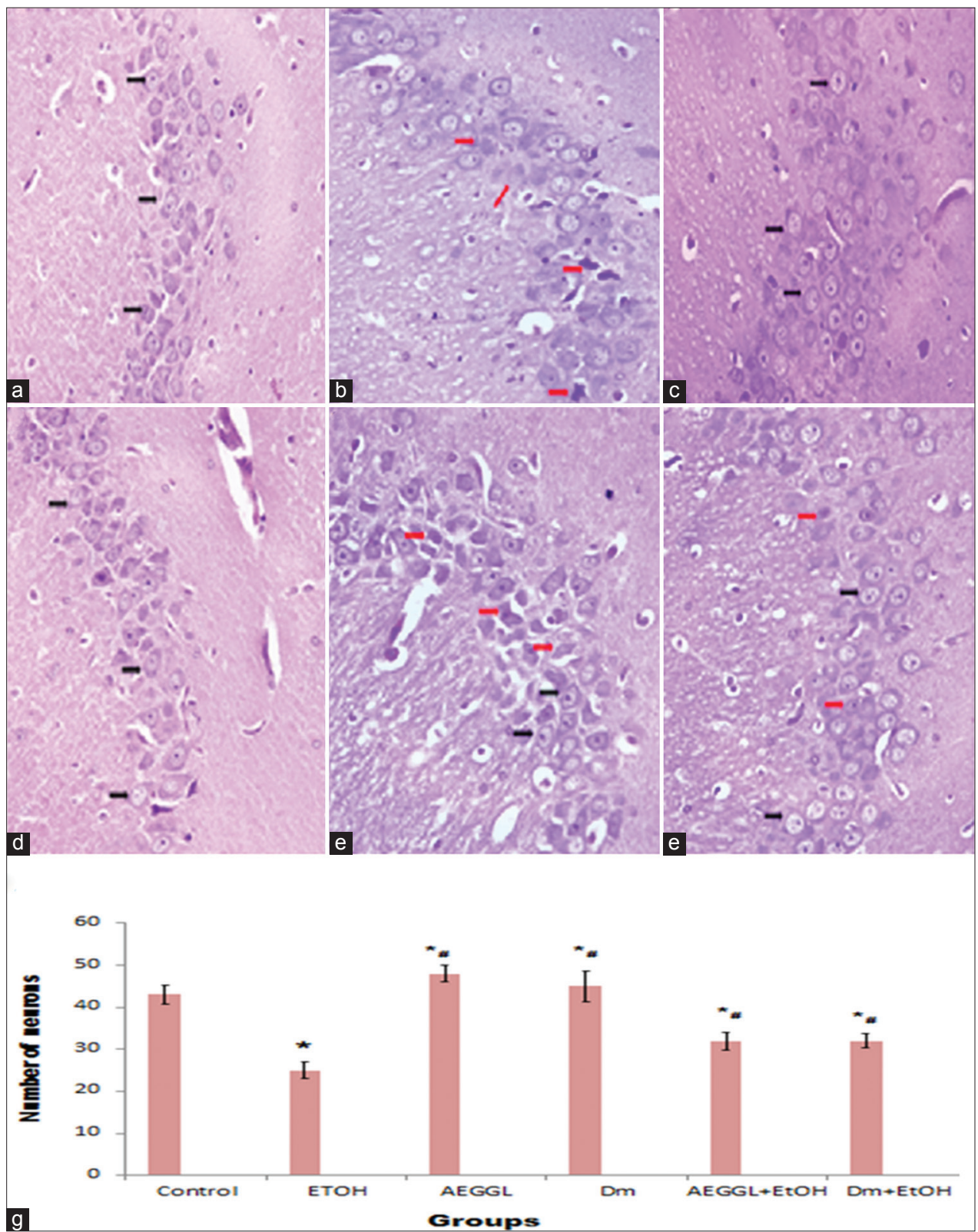

Fig. 10: Effect of enhancement action of Glycyrrhiza glabra Linn. (AEGGL) and diosmetin (Dm) on hippocampal morphology of CA3 pyramidal neurons of rats with ethanol-induced mild cognitive impairment. (a) Control, (b) ETOH, (c) AEGGL, (d) Dm, (e) AEGGL+ETOH,

(f) Dm+ETOH, and (g) total number of neurons in the CA3 region; morphological changes in different experimental groups with hematoxylin-eosin staining data expressed as mean \pm SEM ( $n=6$ and magnification $\times 40)$. *Statistically significant \#no significance

Table 1: Effects of AEGGL and Dm on biochemical parameters

\begin{tabular}{lll}
\hline Groups & AChE $(\mu \mathrm{mol} / \mathrm{min} / \mathbf{m L})$ & BDNF $(\mathrm{pg} / \mathrm{mL})$ \\
\hline Control & $0.53 \pm 0.03$ & $44.3 \pm 2.1$ \\
ETOH & $0.92 \pm 0.04 \#$ & $17.0 \pm 2.4 \#$ \\
AEGGL & $0.52 \pm 0.03^{*}$ & $49.7 \pm 1.3^{*}$ \\
Dm & $0.52 \pm 0.04^{*}$ & $50.0 \pm 2.2^{*}$ \\
AEGGL+EtOH & $0.65 \pm 0.03 \#^{*}$ & $31.8 \pm 1.9 \#^{*}$ \\
Dm+EtOH & $0.68 \pm 0.06 \#^{*}$ & $30.8 \pm 2.3 \#^{*}$ \\
Significance & $\mathrm{F}=14.208 ; \mathrm{P}<0.001$ & $\mathrm{~F}=39.503 ; \mathrm{P}<0.001$ \\
\hline
\end{tabular}

Data were expressed as mean \pm SEM and analyzed by one-way analysis of variance followed by post hoc Tukey's test for multiple comparison of groups ( $\mathrm{n}=6)$. \#is significant from control and "is significant from ETOH. SEM: Standard error mean, AEGGL: Enhancement action of Glycyrrhiza glabra Linn., Dm: Diosmetin, AChE: Acetylcholinesterase the learning, spatial, and reference memory is related with the enhanced level of AChE and decreased BDNF in ETOH group rats. Overall, the biochemical results suggest that the AChE level was increased by ethanol induction as the result of it the Ach level might have decreased. The drug components used are tried to bring back the Ach level to normal by decreasing the ACh esterase level. AEGGL and Dm have the beneficial effects on behavioral and biochemical parameters to enhance the cognitive abilities. Scanty studies are available about potential of Dm on lipophilicity and blood-brain barrier (BBB) permeability. Dm molecule has the high probability to cross BBB and high lipophilicity among the flavonoids and dock with $\mathrm{P}^{38} \mathrm{MAPK}$, c-JNK ERK1/2 signaling pathways in brain [33]. Some in vivo studies exhibit the neurocognitive effects and protective effects of Dm on hippocampal integrity cognitive dysfunctions [34]. Plasma AChE is a marker for Alzheimer's disease 
and associated with cognitive decline in aging [35]. Our experiment biochemical outcome suggests that depressed AChE levels are necessary in retrieval of spatial memory. It is also been inferred that experimentally trained memory is stored in the neocortex and recalled by glutamatergic signals from the prefrontal cortex [36] because of an intercommunication between prefrontal cortex and hippocampus in memory retrieval [37]. Biochemical and pathological studies were conducted titanium nanoparticles induced neurotoxicity in Wistar rats that results correlated with the current study [38]. Another study reported that Hypericum perforatum alleviates the neurotoxicity and AD. Histopathological studies were shown as appeared as shrunken and decreased number of the pyramidal cells [39]. These results inferred with our study and suggest that the herbal plants could be a valuable source of drug against NDD which will prove through highthroughput screening for further drug discovery fields [40]. Our results exhibit that elevated BDNF level in drug-treated group may be implied with prevention of cognitive impairment. Decreased BDNF in ETOH group showed that progressive $\mathrm{MCI}$ for 21 days. BDNF is responsible for neural integrity, synaptic plasticity, and neurogenesis this might be inferred with our AEGGL group rats that they performed well in locomotion and spatial memory [41] even in AEGGL+EtOH group. We could enlighten the BDNF is a potential biomarker which is playing a key role in neuronal survival and differentiation of neuronal population of adulthood $[42,43]$ in ETOH-induced cognitive impairment.

\section{CONCLUSION}

We have observed that AEGGL and Dm prevent the ETOHinduced cognitive impairment in Wistar rats. This might be due to anticholinesterase activity and increased synthesis of BDNF levels in brain. AEGGL and Dm could be used as precursor material to derive potential drug molecule for treating ethanol-induced MCI. Molecular level studies are required to enlighten the exact mechanism of being neuroprotective agent against alcohol-related disorders.

\section{AUTHORS' CONTRIBUTION}

Sasikumar A - conducts the research work, acquisition of data, and interpretation of the work. Suba Malani S - drafting and revising the intellectual work of this study. Manikandan S - conception, design of work, final approval, and communication. Ramaswamy $\mathrm{C}$ - interpretation of data and summarize the work.

\section{CONFLICTS OF INTEREST}

Nil.

\section{REFERENCES}

1. WHO. Country Profile: South-East Asia Region in WHO Global Status Report on Alcohol. Geneva, Switzerland: WHO; 2004. p. 1-5.

2. de la Monte SM, Kril JJ. Human alcohol-related neuropathology. Acta Neuropathol 2014;127:71-90.

3. Haorah J, Ramirez SH, Floreani N, Gorantla S, Morsey B, Persidsky Y, et al. Mechanism of alcohol-induced oxidative stress and neuronal injury. Free Radic Biol Med 2008;45:1542-50.

4. Kim JS, Lee HJ, Kim JC, Kang SS, Bae CS, Shin T, et al. Transient impairment of hippocampus-dependent learning and memory in relatively low-dose of acute radiation syndrome is associated with inhibition of hippocampal neurogenesis. J Radiat Res 2008;49:517-26.

5. Hasanein P, Seifi R, Hajinezhad MR, Emamjomeh A. Rosmarinic acid protects against chronic ethanol-induced learning and memory deficits in rats. Nutr Neurosci 2017;20:547-54.

6. Wattanathorn N, Phunchago S, Muchimapura. Mulberry fruit mitigates alcohol neurotoxicity and memory impairment induced by chronic alcohol intake. Am J Appl Sci 2012;4:484-91.

7. Zorumski CF, Mennerick S, Izumi Y. Acute and chronic effects of ethanol on learning-related synaptic plasticity. Alcohol 2014;48:1-7.

8. Gawel K, Labuz K, Gibula-Bruzda E, Jenda M, MarszalekGrabska M, Filarowska J, et al. Cholinesterase inhibitors, donepezil and rivastigmine, attenuate spatial memory and cognitive flexibility impairment induced by acute ethanol in the barnes maze task in rats. Naunyn Schmiedebergs Arch Pharmacol 2016;389:1059-71.
9. Chen A, Xiong LJ, Tong Y, Mao M. The neuroprotective roles of BDNF in hypoxic ischemic brain injury. Biomed Rep 2013;1:167-76.

10. Li X, Huang M, Zhao R, Zhao C, Liu Y, Zou H, et al. Intravenously delivered allogeneic mesenchymal stem cells bidirectionally regulate inflammation and induce neurotrophic effects in distal middle cerebral artery occlusion rats within the first 7 days after stroke. Cell Physiol Biochem 2018;46:1951-70

11. Ali M, Muhammad S, Shah MR, Khan A, Rashid U, Farooq U, et al. Neurologically potent molecules from Crataegus oxyacantha; isolation, anticholinesterase inhibition, and molecular docking. Front Pharmacol 2017;8:327.

12. Sanjai S. Glycyrrhiza glabra: Medicine over the millennium. Indian J Nat Prod Resour 2005;4:358-67.

13. Chakravarthi KK, Avadhani R. Beneficial effect of aqueous root extract of Glycyrrhiza glabra on learning and memory using different behavioral models: An experimental study. J Nat Sci Biol Med 2013;4:420-5

14. Sharma V, Agrawal RC, Vinoy KS. Assessment of median lethal dose and anti-mutagenic effects of Glycyrrhiza glabra root extract against chemically induced micronucleus formation in Swiss albino mice. Int $\mathbf{J}$ Basic Clin Pharm 2017;2:292-7.

15. Villa P, Dario C, Francesco LD. Amalia G, Giuseppina P, Raffaella P. Protective effect of diosmetin on in vitro cell membrane damage and oxidative stress in cultured rat hepatocytes. Toxicology 1992;73:179-89.

16. de Oliveira DR, Zamberlam CR, Gaiardo RB, Rêgo GM, Cerutti JM, Cavalheiro AJ, et al. Flavones from Erythrina falcata are modulators of fear memory. BMC Complement Altern Med 2014;14:288.

17. Al-Dabbas M, Al-Ismail K, Kitahara K, Tadera K. The effects of different inorganic salts, buffer systems, and desalting of Varthemia crude water extract on DPPH radical scavenging activity. Food Chem 2007;2:734-9.

18. Srikumar MS. Role of Acorus calamus and Alpha asarone on hippocampal dependent memory in noise stress exposed rats. Pak J Biol Sci 2013;16:770-8

19. Bhagya V, Christofer T, Shankaranarayana Rao BS. Neuroprotective effect of Celastrus paniculatus on chronic stress-induced cognitive impairment. Indian J Pharmacol 2016;48:687-93.

20. Goldstein LB, Davis JN. Beam-walking in rats: Studies towards developing an animal model of functional recovery after brain injury. J Neurosci Methods 1990;31:101-7.

21. Ellman GL, Courtney KD, Andres V Jr. Feather-Stone RM. A new and rapid colorimetric determination of acetylcholinesterase activity. Biochem Pharmacol 1961;7:88-95.

22. Haider S, Saleem S, Perveen T, Tabassum S, Batool Z, Sadir S, et al. Age-related learning and memory deficits in rats: Role of altered brain neurotransmitters, acetylcholinesterase activity and changes in antioxidant defense system. Age (Dordr) 2014;36:9653.

23. Koike M, Shibata M, Tadakoshi M, Gotoh K, Komatsu M, Waguri S, et al. Inhibition of autophagy prevents hippocampal pyramidal neuron death after hypoxic-ischemic injury. Am J Pathol 2008;172:454-69.

24. Pu F, Mishima K, Irie K, Motohashi K, Tanaka Y, Orito K, et al. Neuroprotective effects of quercetin and rutin on spatial memory impairment in an 8-arm radial maze task and neuronal death induced by repeated cerebral ischemia in rats. J Pharmacol Sci 2007;104:329-34.

25. de Wit H, Crean J, Richards JB. Effects of d-amphetamine and ethanol on a measure of behavioral inhibition in humans. Behav Neurosci 2000;114:830-7.

26. Dougherty DM, Marsh-Richard DM, Hatzis ES, Nouvion SO, Mathias CW. A test of alcohol dose effects on multiple behavioral measures of impulsivity. Drug Alcohol Depend 2008;96:111-20.

27. Giocomo LM, Hasselmo ME. Nicotinic modulation of glutamatergic synaptic transmission in region CA3 of the hippocampus. Eur $\mathrm{J}$ Neurosci 2005;22:1349-56.

28. Radcliffe KA, Fisher JL, Gray R, Dani JA. Nicotinic modulation of glutamate and GABA synaptic transmission of hippocampal neurons. Ann N Y Acad Sci 1999;868:591-610.

29. Oyinbo CA, Robert FO, Avwioro OG, Igbigbi PS. Jobelyn suppresses hippocampal neuronal apoptosis and necrosis in experimental alcohol-induced brain stress. Pathophysiology 2018; pii: S09284680(18)30001-4.

30. Lim GP, Yang F, Chu T, Gahtan E, Ubeda O, Beech W, et al. Ibuprofen effects on alzheimer pathology and open field activity in APPsw transgenic mice. Neurobiol Aging 2001;22:983-91.

31. Hendricson AW, Miao CL, Lippmann MJ, Morrisett RA. Ifenprodil and ethanol enhance NMDA receptor-dependent long-term depression. J Pharmacol Exp Ther 2002;301:938-44.

32. Vengeliene V, Bilbao A, Molander A, Spanagel R. Neuropharmacology 
of alcohol addiction. Br J Pharmacol 2008;154:299-315.

33. Subamalani S, Sasikumar A. An in-silico study to identify possible novel mitogen activated protein kinases signalling pathway inhibitors from selected natural antioxidants. Int J Pharm Bio Sci 2017;8:195-201.

34. Kumar AS, Malani SS, Ramaswamy C. Morphometric analysis of diosmetin and glabridin reinforces the hippocampal integrity in ethanol induced neuronal damage model rats. Int $\mathrm{J}$ Res Ayurveda Pharm 2017;8:207-10.

35. García-Ayllón MS, Riba-Llena I, Serra-Basante C, Alom J, Boopathy R, Sáez-Valero J, et al. Altered levels of acetylcholinesterase in alzheimer plasma. PLoS One 2010;5:e8701.

36. Hasegawa I, Fukushima T, Ihara T, Miyashita Y. Callosal window between prefrontal cortices: Cognitive interaction to retrieve long-term memory. Science 1998;281:814-8.

37. Preston AR, Eichenbaum H. Interplay of hippocampus and prefrontal cortex in memory. Curr Biol 2013;23:R764-73.
38. Vasantharaja D, Ramalingam V. Neurotoxic effect of titanium dioxide nanoparticles: Biochemical and pathological approach in male Wistar rats. Int J App Pharm 2018;4:74-81.

39. Zerrouki K, Djebli N, Ozkan E, Ozsoy N, Gul O, Mat A. Hypericumperforatum improve memory and learning in alzheimer's model: Experimental study in mice. Int J Pharm Pharm Sci 2016;8:49-57.

40. Roy S, Awasthi H. Herbal medicines as neuroprotective agent: A mechanistic approach. Int J Pharm Pharm Sci 2017;9:1-7.

41. Taliaz D, Stall N, Dar DE, Zangen A. Knockdown of brain-derived neurotrophic factor in specific brain sites precipitates behaviors associated with depression and reduces neurogenesis. Mol Psychiatry 2010;15:80-92.

42. Schinder AF, Poo M. The neurotrophin hypothesis for synaptic plasticity. Trends Neurosci 2000;23:639-45.

43. Huang EJ, Reichardt LF. Neurotrophins: Roles in neuronal development and function. Ann Rev Neurosci 2001;24:677-736. 